Check for updates

Cite this: RSC Adv., 2018, 8, 4006

Received 24th November 2017

Accepted 8th January 2018

DOI: $10.1039 / c 7 r a 12753 g$

rsc.li/rsc-advances

\section{UV and blue-light anti-reflective structurally colored contact lenses based on a copolymer hydrogel with amorphous array nanostructures $\dagger$}

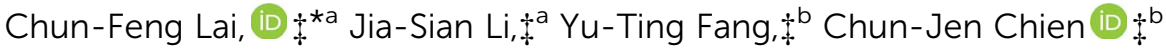 \\ and Chien-Huei Leełc
}

\begin{abstract}
This study presents a facile and inexpensive method for fabricating novel contact lenses with anti-reflective (AR) properties for UV and blue light based on a copolymer hydrogel with amorphous array (AA) nanostructures. The AR structurally colored contact lenses exhibited high optical transparency, homogeneous bluish structural color, and physical rigidity. In addition, the proposed contact lenses blocked short-wavelength blue transmittance by approximately $20 \%$ and had high visible-light transmittance of over $90 \%$. Consequently, the risk of harm posed by commercial contact lenses with chemical dyes was avoided. Adjusting the volume fraction of the silica nanospheres of the AAs in the hydrogel contact lenses was quite effective for controlling the structural colors, transmittance, equilibrium water content (EWC), and oxygen permeability (Dk). The EWC and Dk of the structurally colored contact lenses were in the range of $40.14-45.65 \%$ and $11.6-13.7$ barrers, respectively. These values indicate that the characteristics of the AR structurally colored contact lenses satisfied the requirements of daily disposable contact lenses. Therefore, the proposed AR structurally colored contact lenses are very promising as substitutes for commercial colored contact lenses with chemical dyes.
\end{abstract}

\section{Introduction}

White light-emitting diodes (WLEDs) in solid-state devices have been widely applied for the backlight modules of digital screens, such as TVs, LCDs, tablets, and mobile phones. ${ }^{\mathbf{1 , 2}}$ Commercially available WLEDs generally use blue LEDs to excite yellow phosphor. ${ }^{3}$ Therefore, WLEDs contain high-energy blue light, which is responsible for the blue-light hazard (BLH) problem. A report by the US Department of Energy on BLH discussed how exposure to excessive blue light at approximately 400-480 $\mathrm{nm}$ damages the retina. More and more people are being exposed to high-energy blue light from WLEDs, which has been demonstrated to affect human physiology and psychology. ${ }^{4-6}$ In addition, UV light and strong blue-light radiation damage the human eye and cause diseases, such as cataracts and age-related macular degeneration, by cumulative injury. ${ }^{7,8}$ Soft colored contact lenses are commonly used worldwide because these lenses enable the correction of vision,

${ }^{a}$ Department of Photonics, Feng Chia University, Seatwen, Taichung 40724, Taiwan. E-mail: chunflai@fcu.edu.tw

${ }^{b}$ Section of Medical Device and Cosmetics Analysis, Division of Research and Analysis, Food and Drug Administration, Taipei 11561, Taiwan

${ }^{c}$ Maxinnop Enterprise Co., Ltd., Taipei 105, Taiwan

$\dagger$ Electronic supplementary information (ESI) available. See DOI: $10.1039 / \mathrm{c} 7 \mathrm{ra12753g}$

\$ All authors contributed equally to this work. as well as for fashion and beauty purposes. Therefore, developing colored contact lenses with UV and blue-light antireflective (AR) properties is extremely important. In this study, we propose novel structurally colored contact lenses with UV and blue-light AR properties using amorphous array (AA) nanostructures.

The structural color of photonic structures is generally divided into periodic structures consisting of long-range ordered structures with iridescence (angle-dependent), ${ }^{\mathbf{9} 10}$ and short-range ordered amorphous (or quasi-amorphous) structures with non-iridescence (angle-independent). ${ }^{11-13}$ Structural color is color generated by scattering, refraction, interference, or diffraction of visible light by microstructures or nanostructures. The mechanism of generating structural color differs from those of chemical dyes and pigments. Noniridescent structural colors with unique properties endow AAs with mild, bright and angle-independent characteristics, which have been proposed for application in optical devices. ${ }^{\mathbf{1 4}}$

Generation of a colloidal nanosphere suspension is a facile approach to prepare structurally colored materials with periodic or AA nanostructures. ${ }^{9-13}$ Structural color can be used as an optimal method for fabricating colored contact lenses compared with commercial colored contact lenses because of the bright color and absence of chemical dye in the former. Gu et al. reported two approaches for fabricating structurally colored contact lenses using periodic crystal nanostructures. ${ }^{15,16}$ The iridescent contact lenses exhibited brilliant colors under 
light illumination, but they did not block UV and blue light. Therefore, we propose novel structurally colored contact lenses that are AR for UV and blue light using AA nanostructures. In this study, we report a facile approach for fabricating the proposed colored contact lens by decorating the hydrogels with AAs by UV photo-polymerization in molds. The AR structurally colored contact lenses were fabricated by dispersing silica nanoparticles with AAs in the copolymer hydrogel precursor. Silica nanospheres were used because silica materials are generally recognized as safe by the United States Food and Drug Administration. The obtained AR contact lenses blocked UV and blue light, transmitted over $90 \%$ of visible light, and had a bluish structural color without using chemical dye, which prevented the potential harm from dye leakage posed by commercial colored contact lenses.

Poly(2-hydroxyethyl methacrylate) [poly(HEMA)] and poly(methylmethacrylate) [poly(MMA)] materials are commonly used in soft contact lenses. ${ }^{\mathbf{1 7}}$ HEMA is the host material for soft contact lenses, whereas MMA can increase the hardness and adhesion of hydrogel contact lenses. In addition, $N$-vinylpyrrolidone (NVP) in poly(NVP-co-HEMA) hydrogels have also been widely studied and used as soft contact lenses because of their special molecular structure and ability to improve the water content of the hydrogel. ${ }^{\mathbf{1 8 , 1 9}}$ Copolymerization of monomer MMA with $N, N$-dimethylacrylamide (DMAA) hydrogel has been reported to balance mechanical strength and water content. ${ }^{20}$ Moreover, increased DMAA enhanced the equilibrium water content (EWC) and oxygen permeability (Dk). ${ }^{21,22}$ In this study, the AAs of silica nanospheres were homogeneously dispersed in the hydrogel precursor containing HEMA, MMA, NVP, and DMAA without additional surfactant. The obtained AR structurally colored contact lenses exhibited blue structural color and blocked UV and blue light. The effect was varied by adjusting the volume fraction of silica nanospheres $\left(\phi_{\text {silica }}\right)$. We also investigated the hydrogel material characteristics such as Fourier transform infrared (FT-IR) spectra, optical reflectance and transmittance, EWC, Dk, and contact angle (CA) to demonstrate the validity of this study for application in soft contact lenses. The AR property towards blue light of structurally colored contact lenses has substantial potential for commercial contact lenses, which use chemical dyes to absorb blue light (Fig. S1 and Table S1†).

\section{Experimental section}

\subsection{Materials}

Monomers HEMA, NVP, and DMAA were purchased from Sigma-Aldrich. MMA was purchased from Acros Organics. Ethylene glycol dimethylacrylate (EGDMA) as the crosslinking agent and 2-hydroxy-2-methylpropiophenone (HMPP) as the photoinitiator were purchased from Tokyo Chemical Industry Co., Ltd. The chemical reagents HEMA, MMA, EGDMA, and DMAA were purified by reduced pressure. Absolute ethanol (99.5\%) was purchased from Echo Chem. Co., Ltd. Deionized (DI) water was used in this study. Monodisperse non-uniform silica nanospheres with a mean diameter $\left(D_{\text {mean }}\right)$ of $151 \mathrm{~nm}$ and a polydispersity index of approximately 0.4 were synthesized to form AAs according to a previously reported method (Fig. S2†). ${ }^{13}$

\subsection{Preparation of copolymer hydrogel with silica nanospheres of AAs}

In this study, the copolymer hydrogel precursor mixtures containing HEMA, MMA, NVP, and DMAA monomers were mixed at a certain weight percentage ( $\mathrm{wt} \%$ ) to produce contact lenses with high Dk properties. The mixtures were polymerized in the presence of $1 \mathrm{wt} \%$ EGDMA and $2 \mathrm{wt} \%$ HMPP to yield colorless transparent soft contact lenses (standard, Table S2 $\dagger$ ). Silica nanosphere powders with three different $w \mathrm{t} \%$ were dispersed in $1.0 \mathrm{~g}$ of absolute ethanol using ultrasonication to complete dispersion. Then, suspensions with AAs of silica nanospheres at concentrations of $18.6 \mathrm{wt} \%, 33.8 \mathrm{wt} \%$, and $46.8 \mathrm{wt} \%$ were mixed with the copolymer hydrogel by vortex mixing. Finally, the absolute ethanol in the mixing hydrogel was evaporated by heating the solutions at $50{ }^{\circ} \mathrm{C}$ in a convection oven. The final $\phi_{\text {silica }}$ concentrations of AAs were 10\% (sample A), 20\% (sample $\mathrm{B}$ ), and $30 \%$ (sample $\mathrm{C}$ ). The synthesis parameters are summarized in Table S2. $\dagger$

\subsection{Preparation of the AR structurally colored contact lenses}

A schematic illustration of the preparation of AR structurally colored contact lenses with AA nanostructures in molds is shown in Fig. 1. First, $50 \mu \mathrm{L}$ of hydrogel with AAs was added to the polypropylene (PP) lens mold. Then, the top PP mold was applied carefully onto the bottom PP mold lid. During the pressing process, it was ensured that no air bubbles were produced. After photo-polymerization by UV light $(48 \mathrm{~mW}$ $\mathrm{cm}^{-2}$ ) for $5 \mathrm{~min}$, the top mold was carefully separated from the bottom mold and a solid-state structurally colored contact lens with bright color and good mechanical strength was obtained. Next, the structurally colored contact lens that adhered onto the top mold was hydrated by DI water and heated to $80^{\circ} \mathrm{C}$ until the contact lens fell off from the top mold. Finally, the structurally colored contact lenses were washed and stored in DI water.

\subsection{Characterization}

The morphology of the silica nanospheres and the hydrogel with AAs were characterized using field emission scanning

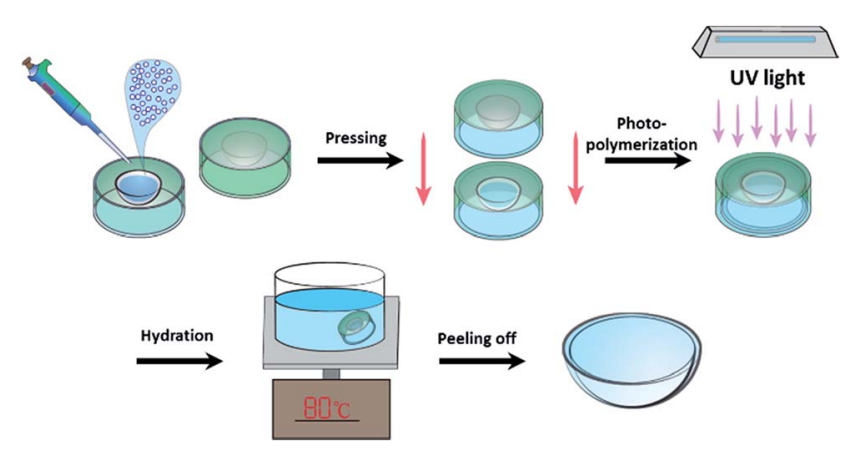

Fig. 1 Schematic illustration of the fabrication of AR structurally colored contact lenses. 
electron microscopy (FESEM) (S-4800; Hitachi) at $3 \mathrm{kV}$. The average diameter of the silica nanospheres was determined by dynamic light scattering using a laser particle size analyzer (LPSA) (N4 plus; Beckman Coulter). FT-IR spectra (Frontier; PerkinElmer Inc.) were measured using attenuated total reflection and recorded with a resolution of $1.0 \mathrm{~cm}^{-1}$. The reflectance and transmittance of the structurally colored contact lenses were measured using a UV-VIS-NIR spectrometer (HR2000; Ocean Optics) and a fiber-coupled UV-enhanced Xe lamp as the white-light source. The contact lenses were soaked in DI water and then mounted on the side surface of the cuvette. The cuvette was placed in a CUV-UV cuvette holder (Ocean Optics) and the measurements were performed at wavelengths ranging from $200 \mathrm{~nm}$ to $1100 \mathrm{~nm}$. The $\mathrm{Dk}$ and EWC of the hydrogel contact lenses were measured according to ISO 183694:2006 (Ophthalmic Optics-Contact lenses-Part 4: Physicochemical properties of contact lens materials) using polarographic analysis and a gravimetric method, respectively. ${ }^{28,29}$ The Dk was analyzed by polarographic analysis using a Model 201T $\mathrm{O}_{2}$ premeometer with a polarographic electrode cell $(8.5 \mathrm{~mm}$ radius; Createch/Rehder Co Dev), temperature and humidity chamber (Model PL-3J; ESPEC Corp.), and motorized thickness meter (Model VL-50; Mitutoyo Corp.). The CAs of the contact lenses with AAs were determined using a CA machine (FTA1000B; first ten angstroms).

\section{Results and discussion}

\subsection{FT-IR analysis of the copolymer hydrogel materials}

Poly(HEMA) is generally used for manufacturing hydrogel contact lenses, but this compound has weak mechanical strength. Therefore, we copolymerized poly(HEMA) with a small amount of MMA to enhance the copolymer hydrogel. MMA can effectively enhance the strength of the gel network and has no effect on the transparency of the gel. NVP can improve the EWC of hydrogel contact lenses. ${ }^{\mathbf{1 8 , 1 9}}$ Hydrophilic DMAA monomers have excellent biocompatibility and good $\mathrm{Dk}^{21,22}$ The integrated poly(HEMA-co-MMA-co-NVP-co-DMAA) copolymer hydrogel was synthesized by free radical copolymerization using EGDMA as the crosslinker and HMPP as the photoinitiator. FT-IR spectra were used to characterize the synthesized copolymer hydrogels of all the produced contact lens samples (Fig. 2 and $\mathrm{S} 3 \dagger$ ). ${ }^{22,23}$

Both samples were characterized using FT-IR to establish the chemical functional groups of all the samples. Table 1 lists the major characteristic peak positions of the functional groups in the standard sample. The standard sample exhibited vibrational bands in the hydroxyl $(\mathrm{O}-\mathrm{H})\left(\sim 3381 \mathrm{~cm}^{-1}\right.$, labelled as A9) and carbonyl $(\mathrm{C}=\mathrm{O})\left(\sim 1719 \mathrm{~cm}^{-1}\right.$, labelled as $\left.\mathrm{A} 7\right)$ stretching regions, stretching of the $\mathrm{C}-\mathrm{N}$ group $\left(\sim 1246 \mathrm{~cm}^{-1}\right.$, labelled as A4), and bands between $1451 \mathrm{~cm}^{-1}$ (labelled as A5) and $2933 \mathrm{~cm}^{-1}$ (labelled as A8) associated with the symmetric and asymmetric $\mathrm{C}-\mathrm{H}$ stretching vibrations $\left(\mathrm{CH}_{2}\right.$ and $\mathrm{CH}_{3}$ groups), respectively. These results show that the carbon functional groups on the membrane-polymer composites can be solely attributed to the presence of the HEMA polymer. ${ }^{23}$ In addition, the peak at $1151 \mathrm{~cm}^{-1}$ (labelled as A3) was caused by the symmetrical $\mathrm{O}-\mathrm{C}$ stretching vibration, which demonstrated the

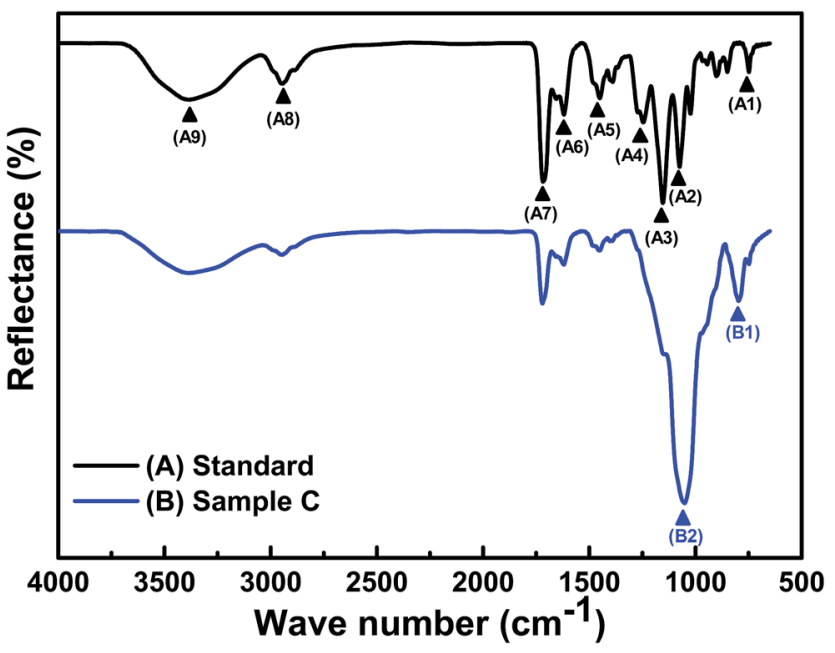

Fig. 2 FT-IR spectra demonstrated the characteristics of the produced contact lens samples.

existence of poly(HEMA-co-MMA-co-NVP-co-DMAA) copolymer segments in the hydrogel contact lenses.

Further, the FT-IR spectrum of sample $\mathrm{C}$ was slightly different from that of the standard sample (Fig. 2). The FT-IR spectra of the calcined silica nanosphere powder is illustrated in Fig. S4. $†$ The strong characteristic peaks at $797 \mathrm{~cm}^{-1}$ (labelled as B1) and $1055 \mathrm{~cm}^{-1}$ (labelled as B2) of sample C were attributed to the $\mathrm{Si}-\mathrm{O}$ asymmetric and symmetric stretching of silica material structures, respectively. ${ }^{24}$ In addition, the samples with different $\phi_{\text {silica }}$ values of AAs were measured by FT-IR spectra (Fig. S3†). Further, it is worth mentioning that the intensity of the peak corresponding to the $\mathrm{Si}-\mathrm{O}$ linkage increases with increasing silica content (Fig. S3†). The results demonstrated that several bands (A1-A4) were covered by the signal of the silica material, demonstrating that these characteristics depend on the $\phi_{\text {silica }}$ of the AAs.

\subsection{Measurement of the optical characteristics of the AR structurally colored contact lenses}

The preparation of the AR structurally colored contact lenses is illustrated in Fig. 1. The internal arrangement of silica nanospheres, which formed the AAs within the hydrogel contact lenses, was observed by FESEM. The FESEM images of sample $\mathrm{C}$

Table 1 Spectral band assignment of standard contact lens sample

Functional group FT-IR peak position $\left[\mathrm{cm}^{-1}\right]$

(A1) C-O-C bending

(A2) $\mathrm{C}-\mathrm{O}-\mathrm{C}$ stretching

748

(A3) C-O-C stretching

1075

(A4) $\mathrm{C}-\mathrm{N}$ stretching

1151

(A5) aliphatic carbon $\left(-\mathrm{CH}_{2}\right)$

(A6) $\mathrm{C}=\mathrm{C}$ stretching $\quad 1620$

(A7) $\mathrm{C}=\mathrm{O}$ stretching 1719

(A8) aliphatic carbon $\left(-\mathrm{CH}_{3},-\mathrm{CH}_{2}\right) \quad 2933$

(A9) O-H stretching 
are shown in Fig. 3. The top surface and cross sections of the structurally colored contact lenses exhibited analogously amorphous crystal structures of silica nanoparticles in the copolymer hydrogel. Characterization of the copolymer hydrogel using FESEM should be performed during the drying process. Therefore, the lattice distances between the silica nanospheres in the FESEM images were shorter than the actual length in the contact lenses. In addition, the 2D fast Fourier transform (FFT) of the FESEM image [inset in Fig. 3(a) and (b)] shows circular and ellipse patterns, respectively. These patterns demonstrate that the AAs were distributed with long-range disordered and short-range ordered structures. Consequently, the angle-independent structural color of the contact lenses was attributed to the isotropic characteristic of the AAs.

The fabricated structurally colored contact lenses demonstrated soft and flexible characteristics after hydration in DI water. Changes in the structural color (from colorless to blue) of the contact lenses are shown and compared in Fig. 4(a). Standard contact lenses are colorless, so are not easy to distinguish when being used. Therefore, commercial standard soft contact lenses add blue dyes into the contact lens hydrogel (Fig. S1†). In this study, bluish contact lenses were easily developed by increasing the $\phi_{\text {silica }}$ of the AAs. When the $\phi_{\text {silica }}$ was increased to $30 \%$ in the copolymer hydrogel, the contact lens of sample C generated bright structural colors [Fig. 4(a)]. The proposed structurally colored contact lenses were brilliant and semi-transparent. Fig. 4(b) and 5(b) exhibit the reflectance and transmittance spectra of the structurally colored contact lenses, respectively. The fabricated lenses were aspherical. Therefore, the reflection spectrum was measured on the top central point of the contact lens with a spot size of $5 \mathrm{~mm}$ diameter. The reflectance measurement was performed using the standard sample before and after hydration as reference for Fig. 4(b) and S5, $\uparrow$ respectively. The reflectance spectra show the distinct weak reflection peak of the three $\phi_{\text {silica }}$ of the structural color contact lenses that were approximately located at $427 \mathrm{~nm}$ (sample A, $\left.\phi_{\text {silica }}=10 \%\right), 435 \mathrm{~nm}\left(\right.$ sample B, $\phi_{\text {silica }}=$ $20 \%$ ), and $463 \mathrm{~nm}$ (sample C, $\phi_{\text {silica }}=30 \%$ ) [Fig. 4(b)]. The reflection peaks could be attributed to the constructive interference of light from the AA nanostructures of the silica nanospheres. The reflection spectra [Fig. 4(b)] of the structurally colored contact lenses reveal a clear reflection peak
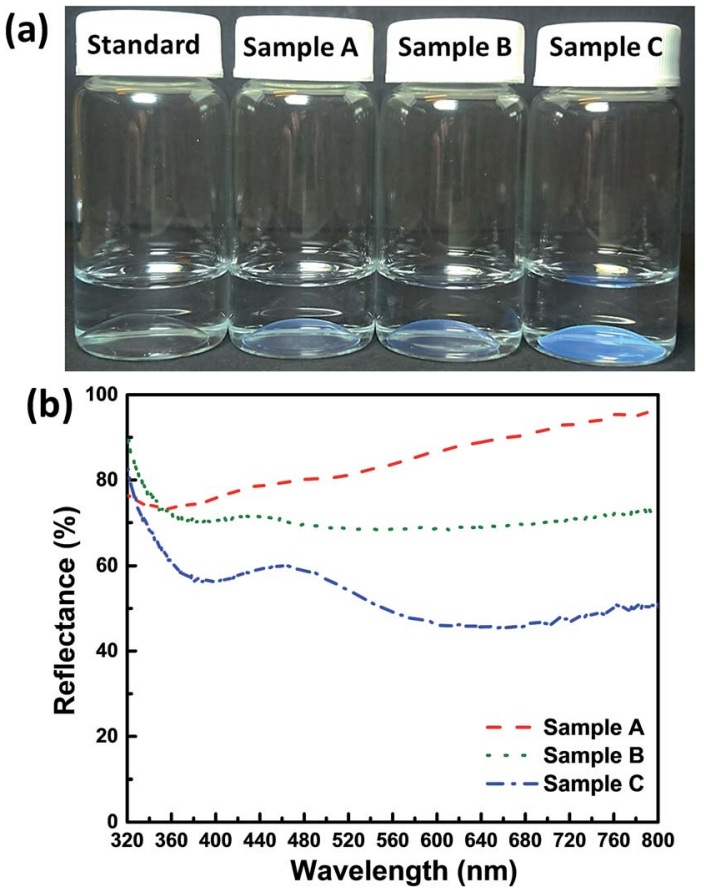

Fig. 4 (a) Digital photographs of all the structurally colored contact lens samples. (b) Reflectance spectra of the three structurally colored contact lenses.

only when $\phi_{\text {silica }}=30 \%$ of silica nanoparticles because the refractive indices of the silica nanospheres and hydrogel were very close. These results are also similar to those in previous reports. ${ }^{15,16}$

In addition, the reflectance and transmittance spectra of sample $\mathrm{C}$ before hydration were measured, and the reflection peak was located at approximately $425 \mathrm{~nm}$ (Fig. S5†). The dominant reflection peak of the sample $\mathrm{C}$ after hydration exhibited a red shift to longer wavelengths, owing to the swelling of copolymer hydrogel in DI water caused by the increased lattice distance between two neighboring silica nanoparticles. Therefore, we calculated the coefficient of expansion of the interlayer space $\left(d_{\text {int }}\right)$ of sample $\mathrm{C}$ before and after the hydration reaction $\left(\phi_{\text {silica }}=30 \%\right)$. The effective refractive index $\left(n_{\text {eff }}\right)$ can be obtained using eqn (1):
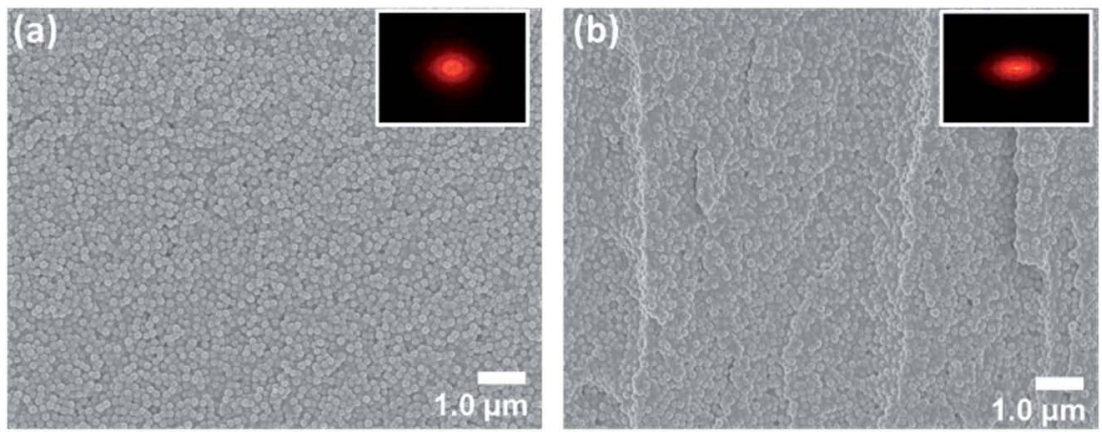

Fig. 3 (a) Top surface and (b) cross-section of FESEM images of the AR structurally colored contact lenses with $\phi_{\text {silica }}=30 \%$ (sample C). Inset shows the 2D FFT images that exhibited long-range disordered and short-range ordered structures. 
(a)

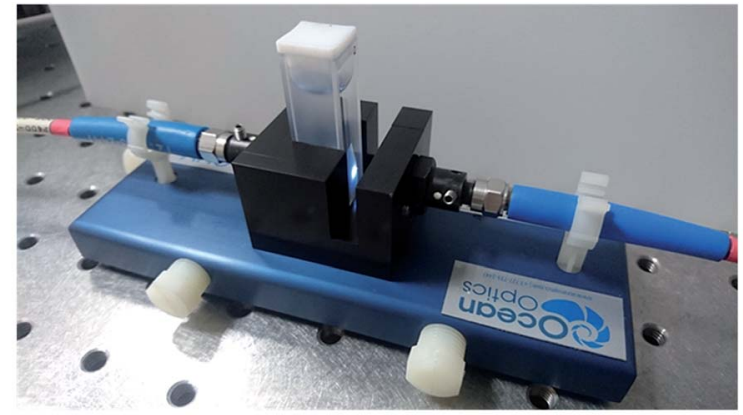

(b)

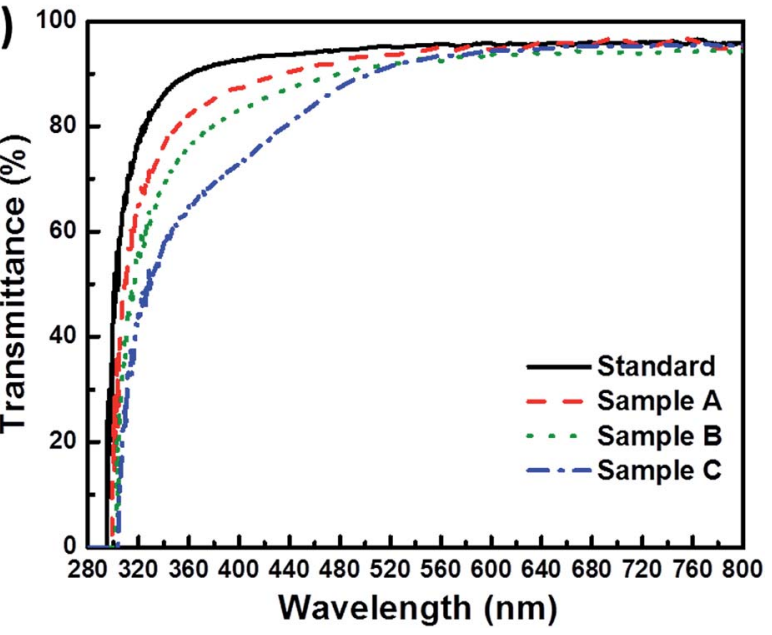

Fig. 5 (a) Digital photograph of the lateral view of the transmittance measurement setup. (b) Transmittance spectra of all the contact lens samples.

$$
n_{\text {eff }}^{2}=n_{\text {silica }}^{2} \phi_{\text {silica }}+n_{\text {hydrogel }}^{2}\left(1-\phi_{\text {silica }}\right)
$$

where $n_{\text {silica }}=1.42$ (ref. 25) and $n_{\text {hydrogel }}=1.43 .^{22}$ In this case, $n_{\text {eff }}=1.427$ for sample $\mathrm{C}\left(\phi_{\text {silica }}=30 \%\right)$. Then, the reflection peak wavelength $\lambda_{\mathrm{R}}$ of the vertical incident was determined according to the Bragg-Snell law [eqn (2)] as follows: ${ }^{\mathbf{1 3 , 1 4}}$

$$
\lambda_{\mathrm{R}}=2 d_{\text {int }} n_{\text {eff }}
$$

Accordingly, the $425 \mathrm{~nm}$ and $463 \mathrm{~nm}$ reflection peak wavelengths (sample C) before and after hydration and $n_{\text {eff }}$ can be calculated using eqn (2) to obtain $d_{\text {int_1 }}=148.9 \mathrm{~nm}$ (before hydration) and $d_{\text {int_2 }}=162.2 \mathrm{~nm}$ (after hydration). The $d_{\text {int } \_1}$ value was very consistent with the $D_{\text {mean }}$ (approximately 151 $\mathrm{nm}$ ) of the results from the LPSA (Fig. S2 $\dagger$ ). Therefore, the coefficient of expansion of the hydrogel for $\phi_{\text {silica }}=30 \%$ is 1.09 in the structurally colored contact lenses before and after hydration. This result is similar to that observed in the periodic crystal structure of contact lenses. ${ }^{15,16}$

In addition, the optical transmittance of all samples was measured using a cuvette (Fig. 5(a)). In this study, blue light is divided into the short-wavelength blue region (SWB, approximately 381-460 $\mathrm{nm}$ ) and the long-wavelength blue region (LWB, approximately 461-500 nm). The transmittances of all contact lens samples are shown in Table 2. The American National Standards Institute of Z80.20 standard has regulated that contact lenses should satisfy Class II UV blocking, which blocks $70 \%$ of UV-A and $95 \%$ of UV-B radiation. Hydrogel contact lenses generally offer little blockage of UV and blue radiation. Therefore, UV absorbers should be added to commercial contact lenses (Fig. S1 and Table S1†) to satisfy the class II UV block. Transmittances for UV-B, UV-A, SWB, LWB, and visible light by the standard lens sample were $29.71 \%, 86.25 \%, 93.16 \%, 94.51 \%$, and $95.05 \%$, respectively. The visible-light transmittance of over $90 \%$ of all contact lenses satisfies the visual requirements, although these measurements also show that the standard hydrogel could not block UV-B and UV-A. By contrast, sample $\mathrm{C}$ with AAs $\left(\phi_{\text {silica }}=\right.$ $30 \%$ ) exhibited optical transmittances of $7.42 \%, 58.24 \%, 76.88 \%$, $87.30 \%$, and $90.12 \%$ for UV-B, UV-A, SWB, LWB, and visible light, respectively. Therefore, sample $\mathrm{C}$ of the structurally colored contact lenses could block $92 \%$ of UV-B and $42 \%$ of UV-A [Fig. 5(b) and Table 2]. In addition, the graded drop in overall transmittance for blue light was estimated to reduce SWB transmittance by approximately $20 \%$ because of the structural color of AAs. This phenomenon is similar to the previously reported AA resin films. ${ }^{\mathbf{1 3 , 1 4}}$ In general, the transmittance decreased as the $\phi_{\text {silica }}$ of AAs increased, and this phenomenon was owing to the strong reflection of AAs. We also performed a transmittance stability test after all samples were stored in DI water for 155 days (Fig. S6†). No differences were found in the samples as shown by the digital photographs in Fig. S6(a). $\dagger$ However, all samples exhibited a smallest transmittance drop of $5 \%$ after storage for 155 days (Table 2). These results demonstrated the high stability of the structurally colored contact lenses.

\begin{tabular}{|c|c|c|c|c|c|}
\hline$T[\%]$ & UV-B (280-315 nm) & UV-A (316-380 nm) & SWB $(381-460 \mathrm{~nm})$ & LWB $(461-500 \mathrm{~nm})$ & Visible $(381-780 \mathrm{~nm})$ \\
\hline Standard & $29.71 \%$ & $86.25 \%$ & $93.16 \%$ & $94.51 \%$ & $95.05 \%$ \\
\hline Sample A & $18.22 \%$ & $77.05 \%$ & $88.88 \%$ & $92.50 \%$ & $93.69 \%$ \\
\hline Sample B & $11.98 \%$ & $70.01 \%$ & $85.07 \%$ & $90.01 \%$ & $91.50 \%$ \\
\hline Sample C & $7.42 \%$ & $58.24 \%$ & $76.88 \%$ & $87.30 \%$ & $90.12 \%$ \\
\hline \multicolumn{6}{|c|}{ All the samples were stored in DI water for 155 days } \\
\hline Standard & $26.69 \%$ & $85.35 \%$ & $92.83 \%$ & $93.70 \%$ & $94.08 \%$ \\
\hline Sample A & $16.53 \%$ & $72.05 \%$ & $83.77 \%$ & $88.95 \%$ & $91.76 \%$ \\
\hline Sample B & $11.34 \%$ & $67.54 \%$ & $81.29 \%$ & $86.96 \%$ & $89.65 \%$ \\
\hline Sample C & $7.37 \%$ & $57.32 \%$ & $73.00 \%$ & $82.65 \%$ & $88.20 \%$ \\
\hline
\end{tabular}

Table 2 Optical transmittance of all the contact lens samples 


\subsection{Measurement of the characteristics of the AR structurally} colored contact lenses

The contact lenses have two unique characteristics, namely EWC and Dk, which should be measured, because they demonstrate the properties of the hydrogel material. EWC was measured using thermogravimetry to evaluate the water content of the contact lenses. ${ }^{26}$ Thermogravimetry was also used to determine the ratio of free-to-bound water in the hydrogel material. The EWC of the standard sample was measured to be $54.70 \%$. Sample A ( $\phi_{\text {silica }}=$ $10 \%$ ) showed an average EWC of $45.65 \%$, indicating that the AAs reduced the EWC. By contrast, the EWCs of samples B ( $\phi_{\text {silica }}=$ $20 \%)$ and $\mathrm{C}\left(\phi_{\text {silica }}=30 \%\right)$ were $39.66 \%$ and $40.14 \%$, respectively. These results demonstrated that AAs increased proportionally with the decrease in EWC. A comparison of the changes in the EWC of each sample is shown in Table 3. Specialists on contact lenses have recommended that the EWC of contact lenses should ideally range within 30 to $40 \%$. EWC of more than $50 \%$ is high for contact lenses, and this characteristic facilitates wear and dry eyes, which lead to discomfort. ${ }^{27}$

In addition, the Dks of all samples were determined by measuring four different thicknesses of stacked contact lens samples to obtain multiple thicknesses (Fig. 6). Dk was derived by measuring the reading for each thickness. The process was repeated to provide the number of results shown in Fig. 6. Prior to the Dk measurements, all contact lens samples were required to be placed in a chamber to be stabilized at $35 \pm 0.5^{\circ} \mathrm{C}$ with an achievable humidity value of approximately $98 \%$. The contact lens was carefully placed on the polarographic electrode cell

Table 3 Dks and EWCs of all the contact lens samples

\begin{tabular}{llll}
\hline Sample & $\mathrm{Dk}^{a}[$ barrers] & $R^{2}$ & EWC [\%] \\
\hline Standard & 16.9 & 0.9978 & $54.70 \%$ \\
Sample A & 13.7 & 0.9986 & $45.65 \%$ \\
Sample B & 11.5 & 0.9995 & $39.66 \%$ \\
Sample C & 11.6 & 0.9914 & $40.14 \%$
\end{tabular}

${ }^{a}$ Conventional Dk units $\left(\times 10^{-11}\left(\mathrm{~cm}^{2} \mathrm{~s}^{-1}\right)\left[\mathrm{ml} \mathrm{O}_{2}\left(\mathrm{ml} \times \mathrm{mm} \mathrm{Hg}^{-1}\right]\right)\right.$, also known as barrers. and during the process it was ensured that no air bubbles were trapped between the contact lens and the electrode cell. The thicknesses of all samples were measured and the results were used to analyze and calculate the Dk. The measured Dks were applied to correct for the effects of the boundary-layer and edge. ${ }^{28,29}$ The measured Dk values of all contact lens samples are also summarized in Table 3. The measurement results exhibited that the Dk of the standard sample is 16.9 barrers, which is similar to that of a general hydrogel contact lens. ${ }^{27}$ In addition, the Dk increased proportionally with increasing EWC. The structurally colored contact lens sample $\mathrm{C}$ had an EWC of approximately $40.14 \%$ and a Dk of approximately 11.6 barrers. Slight differences were found between the EWC and Dk of samples B and C. To confirm this result, we measured the Dk of five random samplings from samples B and C. The findings were measured and confirmed by the Taiwan Food and Drug Administration. The measured results showed that the true percent relative error and the $R^{2}$ of these samples were approximately $1 \%$ and over 0.99 , respectively. Therefore, the Dk of the structurally colored contact lenses could not depend on the $\phi_{\text {silica }}$ of the AAs. The viscosity of the silica-hydrogel suspensions depended on the $\phi_{\text {silica }}$ concentration. Silicahydrogel suspensions with $\phi_{\text {silica }}$ of over $30 \%$ were not used to prepare the samples because of the higher viscosity. Hence, $\phi_{\text {silica }}$ of $30 \%$ is the optimal parameter for the silica-hydrogel suspensions in this study. The hydrogel contact lenses approached the point where the oxygen needs of the cornea would be satisfied. ${ }^{30}$ These results indicate the good performance of the developed structurally colored contact lenses for daily disposable contact lenses.

\subsection{Contact angle measurements of AR structurally colored contact lenses}

The standard sample was sticky compared with the structurally colored contact lenses (samples A-C). Therefore, we inferred that the surface roughness of the contact lenses affected their hydrophilicity, and this supposition is based on previous reports. ${ }^{\mathbf{1 3 1 4}}$ Fig. 7(a) and (b) show the variation in the water CA of the contact lenses with and without AA nanostructures. The (a)

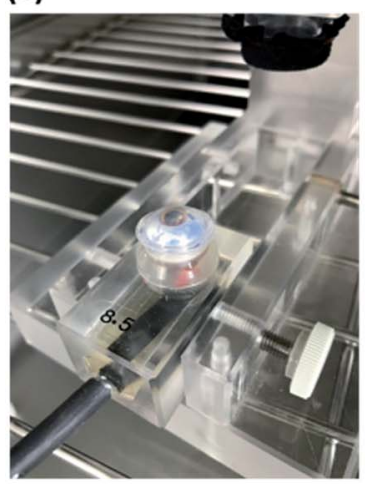

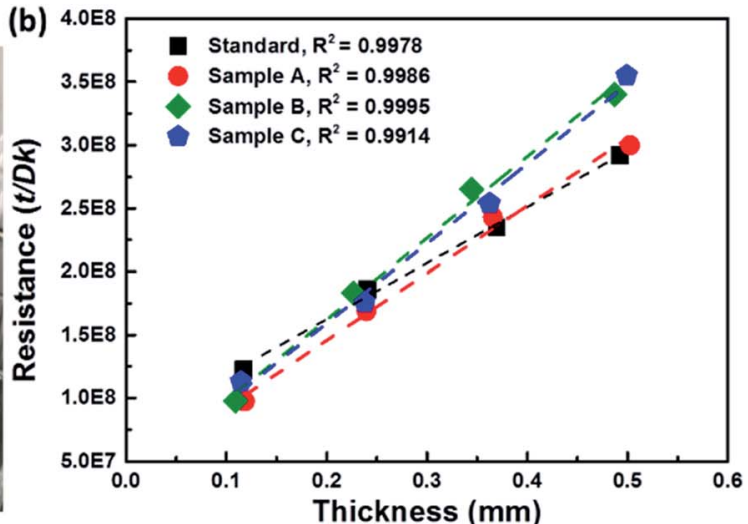

Fig. 6 (a) Polarographic electrode cell for measuring the Dk of sample C. (b) Corrected resistance versus thickness plots for all contact lens samples. The plots are closely approximated by a line $\left(R^{2}>0.99\right)$. 

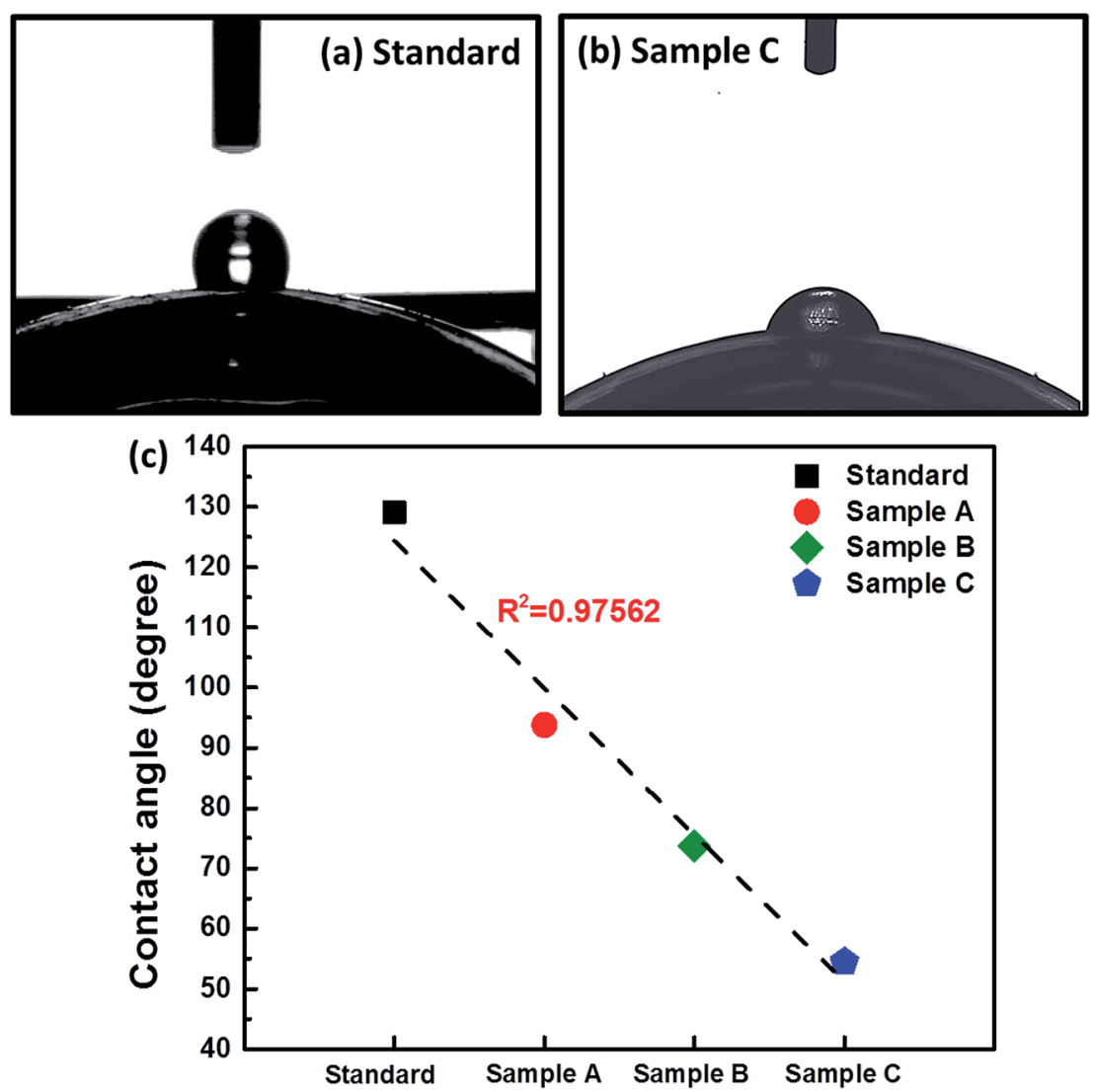

Fig. 7 Optical photographs of (a) standard and (b) sample C for CA measurements. (c) CA versus samples plot closely approximated by a line $\left(R^{2}>\right.$ 0.97).

CA of the standard contact lens was approximately $129.05^{\circ}$ with a hydrophobic surface, which was reduced to nearly $54.42^{\circ}$ with $\phi_{\text {silica }}=30 \%$ (sample C) with a hydrophilic surface. These results indicate that the AA-induced hydrophilic structurally colored contact lenses were successfully obtained in the interfaces between each silica nanosphere. Moreover, the CA on sample A was approximately $93.77^{\circ}$ and reduced to approximately $54.42^{\circ}$ when the $\phi_{\text {silica }}$ was increased [Fig. 7(c)]. The CA showed decreased dependence on the $\phi_{\text {silica }}$ of AAs, because the surface roughness of AAs was greater than that of smooth contact lenses. This result was attributed to the AA surface roughness and this finding is consistent with the previously reported resin films with AA nanostructures. ${ }^{13,14}$ Finally, we could not evaluate the practical usage of these contact lenses because these soft contact lenses are medical devices. Consequently, they must undergo human clinical trials and be approved by the United States Food and Drug Administration before marketing. Therefore, this study only proposed a novel method and demonstrated that contact lenses with AA nanostructures present improved hydrophilicity, are non-sticky, and block UV and blue light without further processing.

\section{Conclusions}

In summary, we presented novel AR structurally colored contact lenses with AA nanostructures. The proposed contact lenses not only blocked UV and blue light under sunlight but also avoided the risk of harm posed by commercial contact lenses colored with chemical dyes. The AR structurally colored contact lenses exhibited homogeneous bluish structural colors and high transmittance of over $90 \%$. Moreover, the proposed contact lenses blocked SWB transmittance by approximately $20 \%$. Adjusting the $\phi_{\text {silica }}$ in the hydrogel contact lenses effectively controlled the structural colors, transmittance, EWC, Dk, and CA. The EWC and Dk of the structurally colored contact lenses were in the range of $40.14-45.65 \%$ and $11.6-13.7$ barrers, respectively, indicating that the structurally colored contact lenses satisfied the requirements for daily disposable contact lenses. Therefore, the AR structurally colored contact lenses are very promising as substitutes for commercial colored contact lenses using chemical dyes.

\section{Conflicts of interest}

There are no conflicts to declare.

\section{Acknowledgements}

This work is supported by the Ministry of Science and Technology (MOST) in Taiwan under contract numbers MOST105-2221-E035-041 and MOST106-2221-E-035-076. The authors appreciate the Precision Instrument Support Center of Feng Chia University for providing the fabrication and measurement facilities. 


\section{References}

1 E. Jang, S. Jun, H. Jang, J. Lim, B. Kim and Y. Kim, WhiteLight-Emitting Diodes with Quantum Dot Color Converters for Display Backlights, Adv. Mater., 2010, 22, 3076.

2 R. J. Xie, N. Hirosaki and T. Takeda, Wide Color Gamut Backlight for Liquid Crystal Displays Using Three-Band Phosphor-Converted White Light-Emitting Diodes, Appl. Phys. Express, 2009, 2, 022401.

3 S. Ye, F. Xiao, Y. X. Pan, Y. Y. Ma and Q. Y. Zhang, Phosphors in phosphor-converted white light-emitting diodes: recent advances in materials, techniques and properties, Mater. Sci. Eng. R Rep, 2010, 71, 1.

4 G. C. Brainard, J. P. Hanifin, J. M. Greeson, B. Byrne, G. Glickman, E. Gerner and M. D. Rollag, Action Spectrum for Melatonin Regulation in Humans, J. Neurosci., 2001, 21, 6405.

5 S. M. Pauley, Lighting for the human circadian clock: recent research indicates that lighting has become a public health issue, Med. Hypotheses, 2004, 63, 588.

6 J. P. Hanifin, K. T. Stewart, P. Smith, R. Tanner, M. Rollag and G. C. Brainard, High-intensity red light suppresses melatonin, Chronobiol. Int., 2006, 23, 251.

7 E. Chamorro, C. B. Arias, M. J. P. Carrasco, J. M. d. Luna, D. Vazquez and C. S. Ramos, Effects of Light-emitting Diode Radiations on Human Retinal Pigment Epithelial Cells In Vitro, Photochem. Photobiol., 2013, 89, 468.

8 U. Wihlmarka, A. Wrigstada, K. Robergb, S. E. G. Nilssona and U. T. Brunkb, Lipofuscin Accumulation in Cultured Retinal Pigment Epithelial Cells Causes Enhanced Sensitivity to Blue Light Irradiation, Free Radical Biol. Med., 1997, 22, 1229.

9 (a) H. S. Lee, T. S. Shim, H. Hwang, S. M. Yang and S. H. Kim, Colloidal Photonic Crystals toward Structural Color Palettes for Security Materials, Chem. Mater., 2013, 25, 2684; (b) Y. Takeoka, M. Honda, T. Seki, M. Ishii and H. Nakamura, Structural Colored Liquid Membrane without Angle Dependence, ACS Appl. Mater. Interfaces, 2009, 1, 982.

10 F. Li, B. Tang, S. Wu and S. Zhang, Facile Synthesis of Monodispersed Polysulfide Spheres for Building Structural Colors with High Color Visibility and Broad Viewing Angle, Small, 2017, 13, 1602565.

11 Y. Takeoka, S. Yoshioka, A. Takano, S. Arai, K. Nueangnoraj, H. Nishihara, M. Teshima, Y. Ohtsuka and T. Seki, Production of Colored Pigments with Amorphous Arrays of Black and White Colloidal Particles, Angew. Chem., Int. Ed., 2013, 52, 7261.

12 (a) D. Ge, L. Yang, G. Wu and S. Yang, Angle-independent colours from spray coated quasi-amorphous arrays of nanoparticles: combination of constructive interference and Rayleigh scattering, J. Mater. Chem. C, 2014, 2, 4395; (b) K. Katagiri, Y. Tanaka, K. Uemura, K. Inumaru, T. Seki and Y. Takeoka, Structural color coating films composed of an amorphous array of colloidal particles via electrophoretic deposition, NPG Asia Mater., 2017, 9, e355.
13 C. F. Lai, Y. C. Wang and H. C. Hsu, High transparency in the structural color resin films through quasi-amorphous arrays of colloidal silica nanospheres, J. Mater. Chem. C, 2016, 4, 398.

14 C. F. Lai, J. S. Li and C. W. Shen, High-Efficiency Robust FreeStanding Composited Phosphor Films with 2D and 3D Nanostructures for High-Power Remote White LEDs, ACS Appl. Mater. Interfaces, 2017, 9, 4851.

15 Z. Xie, L. Li, P. Liu, F. Zheng, L. Guo, Y. Zhao, L. Jin, T. Li and Z. Gu, Self-assembled coffee-ring colloidal crystals for structurally colored contact lenses, Small, 2015, 11, 926.

16 P. Liu, Z. Xie, F. Zheng, Y. Zhao and Z. Gu, Surfactant-free HEMA crystal colloidal paint for structural color contact lens, J. Mater. Chem. B, 2016, 4, 5222.

17 P. C. Nicolson and J. Vogt, Soft contact lens polymers: an evolution, Biomaterials, 2001, 22, 3273.

18 Y. C. Lai, Effect of crosslinkers on photocopolymerization of $N$-vinylpyrrolidone and methacrylates to give hydrogels, $J$. Appl. Polym. Sci., 1997, 66, 1475.

19 Y. C. Lai, A novel crosslinker for UV copolymerization of $\mathrm{N}$ vinyl pyrrolidone and methacrylates to give hydrogels, $J$. Polym. Sci., Part A: Polym. Chem., 1997, 35, 1039.

20 L. M. Muratore and T. P. Davis, Self-reinforcing hydrogels comprised of hydrophobic methyl methacrylate macromers copolymerized with $\mathrm{N}, \mathrm{N}$-dimethylacrylamide, J. Polym. Sci., Part A: Polym. Chem., 2000, 38, 810.

21 T. H. Kim and A. Y. Sung, Preparation and Physical Properties of Hydrogle Lens Containing $\mathrm{N}, \mathrm{N}$ Dimethylacrylamide, J. Korean Chem. Soc., 1994, 54, 761.

22 A. Y. Sung and T. H. Kim, Optical application of poly(HEMAco-MMA) containing silver nanoparticles and $\mathrm{N}, \mathrm{N}$ dimethylacrylamide, Korean J. Chem. Eng., 2012, 29, 686.

23 T. S. Perova, J. K. Vij and H. Xu, Fourier transform infrared study of poly(2-hydroxyethyl methacrylate) PHEMA, Colloid Polym. Sci., 1997, 275, 323.

24 K. Zhang, L. M. Gan, C. H. Chew and L. H. Gan, Silica from hydrolysis and condensation of sodium metasilicate in bicontinuous microemulsions, Mater. Chem. Phys., 1997, 47, 164.

25 Y. L. Ko, H. P. Tsai, K. Y. Lin, Y. C. Chen and H. Yang, Reusable macroporous photonic crystal-based ethanol vapor detectors by doctor blade coating, J. Colloid Interface Sci., 2017, 487, 360.

26 N. Efron and N. A. Brennan, The clinical relevance of hydrogel lens water content, International Contact Lens Clinic, 1987, 10, 9.

27 P. C. Nicolson and J. Vogt, Soft contact lens polymers: an evolution, Biomaterials, 2001, 22, 3273.

28 W. J. Benjamin and Q. A. Cappelli, Oxygen Permeability (Dk) of Thirty-Seven Rigid Contact Lens Materials, Optom. Vis. Sci, 2002, 79, 103.

29 M. D. Young and W. J. Benjamin, Oxygen Permeability of the Hypertransmissible Contact Lenses, Eye Contact Lens, 2003, 29, S17.

30 N. Efron, N. A. Brennan and B. A. Holden, The oxygen permeability of rigid gas permeable contact lens materials: reaching a concensus, Int. Contact Lens Clin., 1987, 10, 62. 